\title{
Sliding Mode Observer and Control Design with Adaptive Parameter Estimation for a Supersonic Flight Vehicle
}

\author{
M. Bahrami, ${ }^{1}$ B. Ebrahimi, ${ }^{2}$ and G. R. Ansarifar ${ }^{1}$ \\ ${ }^{1}$ Department of Mechanical Engineering, Amirkabir University of Technology, Tehran, Iran \\ ${ }^{2}$ Aerospace Research Institute, Ministry of Science Research and Technology, Tehran, Iran \\ Correspondence should be addressed to B. Ebrahimi, ebrahimi@ari.ac.ir
}

Received 4 October 2009; Revised 26 January 2010; Accepted 6 June 2010

Academic Editor: D. Ghose

Copyright ( $) 2010$ M. Bahrami et al. This is an open access article distributed under the Creative Commons Attribution License, which permits unrestricted use, distribution, and reproduction in any medium, provided the original work is properly cited.

\begin{abstract}
Design and synthesis of a nonlinear generic supersonic flight vehicle longitudinal dynamics control for angle-of-attack, AOA, output tracking in the atmospheric flight is presented based on sliding mode control. A sliding mode observer is invoked to estimate AOA which is difficult to measure in practice. Large parameter uncertainties accommodation envisaged by designing adaptive mechanisms for both the control and observer and high chattering authority due to large deviations of aerodynamic coefficients arising from wind-tunnel measurements are inhibited. The employed method enables the sliding mode control design to exhibit the desired dynamic properties during the entire output-tracking process. Simulations results are presented to demonstrate the performance, robustness, and stability.
\end{abstract}

\section{Introduction}

Tail-controlled skid-to-turn flight vehicles are commonly agile systems that should be equipped with control systems whose tasks are to provide stability, disturbance attenuation, and reference signal tracking, while their aerodynamic coefficients vary over a wide dynamic range due to large Mach-altitude fluctuations and due to aerodynamic coefficient uncertainties resulting from inaccurate wind-tunnel measurements.

It is common practice, when designing a control system for a nonlinear flight vehicle, to represent the flight envelope by a grid of Mach-altitude operating points and then to perform a linearization of the nonlinear state equations at trim points of the gridded flight envelope. The plant in fact becomes a differential inclusion under continuously varying flight conditions. There are many possible ways of dealing with the control of such linear and time varying plants. The classical approach is to design a controller for a certain point and then to schedule the controller's gain to place eigenvalues deep in the left half plane and the near real-axis according to a measured or derived parameters that represent flight conditions, such as angle of attack or Mach number [1]. In another method, $\mathrm{H}_{\infty}$ methods are invoked to design a collection of controllers, where, for each operating point in the flight envelope grid, a controller with a fixed structure results [2]. The ensuing set of controllers is then transformed to a single gain scheduled controller by obtaining a leastsquare fit of its parameters with respect to angle of attack, or Mach number, and so forth. For highly agile air vehicles, these techniques would result in an extensive number of controller design points to be able to cope with the drastically changing dynamic behavior throughout the flight envelop.

All of the aforementioned methods are linear design techniques that require either exact knowledge of system parameters or, alternatively, assumption of some uncertainty model such as norm boundedness, thus allowing for robust controller design. On the other hand, sliding mode control may, in principle, be implemented for dynamic systems having only a qualitative description and a number of inequality restrictions. As will be shown in sequel, this approach will provide a simple and high-performance controller on nonlinear system using straightforward design procedure.

Sliding modes are the primary form of operational variable structure systems. A sliding mode is a motion on a discontinuity set of a dynamic system and is characterized by a suite of feedback control law and a decision rule known as switching function. Such modes are used to maintain 
TABle 1: Aerodynamic Polynomial Coefficients.

\begin{tabular}{lc}
\hline$\beta_{1 n}=19.3734$ & $\beta_{1 m}=40.4396$ \\
$\beta_{2 n}=31.0225$ & $\beta_{2 m}=-64.0147$ \\
$\beta_{3 n}=-9.717$ & $\beta_{3 m}=2.9221$ \\
$d_{n}=-1.9481$ & $d_{m}=-11.8029$ \\
\hline
\end{tabular}

the given constraints with utmost accuracy. The sliding mode controller is an attractive robust control algorithm because of its inherent insensitivity and robustness to plant uncertainties and external disturbances.

The nonlinear pitch dynamics of a hypothetical tailcontrolled flight vehicle is derived in Section 2. In Section 3 sliding manifold is employed to control pitch-axis dynamics of the air vehicle. Adaptive sliding mode control is designed in Section 4. Sliding mode observer is designed and utilized in control scheme in Section 5 on the basis of pitch rate measurement for the nonlinear system to estimate angle-ofattack which is difficult to measure in practice. Integration of sliding mode control with adaptation mechanism based on the designed observer is presented in Section 6.

\section{Problem Formulation}

The missile model employed in this analysis is based on the hypothetical tail-controlled air vehicle which has been used as a benchmark in a number of recent studies on nonlinear design techniques [1-3]. The control objective is to force the air vehicle to track a desired motion path generated by the guidance-navigation system as the reference angle-of attack. The problem is first formulated and performance objectives are specified.

2.1. Dynamic Model of the Airframe. The model assumes constant mass, no roll rate, zero roll angle, no side slip, and no yaw rate. Under these assumptions, the longitudinal nonlinear equation of motion is reduced to two forces and one moment. Using body axis coordinates, Figure 1, these equations are

$$
\begin{aligned}
& F_{x}=Q S C_{D}, \\
& F_{z}=Q S C_{N}, \\
& M_{y}=Q S d C_{M} .
\end{aligned}
$$

The dynamic pressure is defined as

$$
Q=\frac{1}{2} \rho V_{m}^{2}=0.7 P_{0} M^{2}
$$

Note that velocity and air density are not assumed to be constant or slow-varying, but a standard atmospheric model is assumed in simulation based on previously reported data [4].

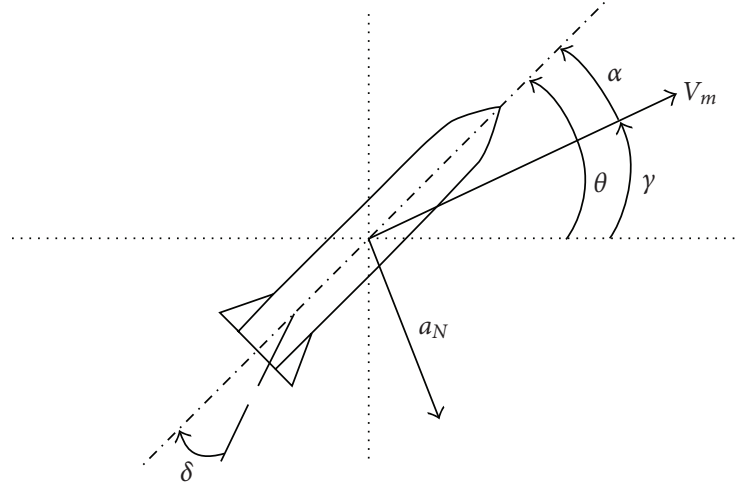

FIgURE 1: Airframe and dynamic variables.

Aerodynamic polynomials resulting from wind-tunnel measurements are given as [1]

$$
\begin{aligned}
C_{D} & =-0.3, \\
C_{N}(\alpha, \delta, M) & =\beta_{1 n} \alpha^{3}+\beta_{2 n} \alpha|\alpha|+\beta_{3 n}\left(2-\frac{M}{3}\right) \alpha+d_{n} \delta \\
& =C_{n}(\alpha, M)+d_{n} \delta, \\
C_{M}(\alpha, \delta, M) & =\beta_{1 m} \alpha^{3}+\beta_{2 m} \alpha|\alpha|+\beta_{3 m}\left(-7+\frac{8}{3} M\right) \alpha+d_{m} \delta \\
& =C_{m}(\alpha, M)+d_{m} \delta .
\end{aligned}
$$

The numerical values of the various constant parameters in the dynamic equations are given in Table 1 . Note that all coefficients are dimensionless and all angles are in radians.

Consequently, the differential equations describing the body motion are

$$
\begin{aligned}
& \dot{\alpha}=\frac{\cos \alpha}{m V_{m}} F_{z}+q, \\
& \dot{q}=\frac{M_{y}}{I_{y}}, \\
& \dot{V}_{m}=\frac{F_{x} \cos \alpha}{m}-\left|\frac{F_{z} \sin \alpha}{m}\right|, \\
& \dot{h}=M v_{s} \sin \gamma, \\
& \dot{X}=M v_{s} \cos \gamma .
\end{aligned}
$$

The tail-fin actuator dynamics describing the tail deflection is

$$
\dot{\delta}=\frac{1}{\tau_{a}}\left(\delta_{c}-\delta\right),
$$

where actuator fin deflection is limited to $-40^{\circ} \leq \delta \leq 40^{\circ}$. Table 2 gives the flight vehicle constants.

As shown in the equations of motion, the time-varying aerodynamic parameters contribute heavily to the variation of dynamic forces and moments exerted on the vehicle airframe. It is assumed here as is often customary in 
TABLE 2: Flight Vehicle Constants.

\begin{tabular}{lc}
\hline Symbol & Quantity \\
\hline$I_{y}$ & $247.43662 \mathrm{~kg} \mathrm{~m}^{2}$ \\
$d$ & $0.2286 \mathrm{~m}$ \\
$S$ & $0.0409 \mathrm{~m}^{2}$ \\
$m$ & $204.108 \mathrm{~kg}$ \\
$\tau_{a}$ & $1 / 150 \mathrm{~s}$ \\
$g$ & $9.81 \mathrm{~m} / \mathrm{s}^{2}$ \\
\hline
\end{tabular}

air vehicle autopilot design that the vehicle velocity (or Mach number) is constant and the nonlinear state equation associated with $V_{m}$ is dropped from the design model [1]. In this case, the vehicle velocity becomes an independent (external) parameter upon which the state dynamics depend.

By rewriting (4) and (5), it is easy to show that

$$
\begin{aligned}
& \dot{\alpha}(t)=K_{\alpha} M C_{N}[\alpha(t), \delta(t)] \cos \alpha(t)+q(t), \\
& \dot{q}(t)=K_{q} M^{2} C_{M}[\alpha(t), \delta(t)], \\
& \dot{\delta}(t)=\frac{1}{\tau_{a}}\left[\delta_{c}(t)-\delta(t)\right],
\end{aligned}
$$

where $K_{\alpha}=0.7 P_{0} S / m v_{s}, K_{q}=0.7 P_{0} S d / I_{y}$. Numerical values of $K_{\alpha}$ and $K_{q}$ are $0.0206 \mathrm{~s}^{-1}$ and $1.2326 \mathrm{~s}^{-2}$, respectively, at an altitude of $6100 \mathrm{~m}$.

2.2. Performance Objective. The control objective is to force the air vehicle to track a desired motion path. To this end, the guidance-navigation system generates the reference angle-ofattack, denoted by $\alpha_{d}$. This reference signal is compared with the actual signal measured in order to reveal a tracking error. The control problem consists of generating a tail deflection $\delta_{c}$ that corresponds to the required maneuver. Considering the air vehicle flying at an altitude of $6100 \mathrm{~m}(\approx 20,000 \mathrm{ft})$, the closed-loop system should maintain stability over the operating range.

\section{Control Design}

The longitudinal model of the flight vehicle described by (10) is a special case of a general nonlinear system of the form

$$
\begin{gathered}
\dot{x}=f(x)+g(x) u, \\
y=h(x),
\end{gathered}
$$

where $f, g$, and $h$ are sufficiently smooth functions.

Remark 1. Relative degree, $r$ for nonlinear system (11), is defined as follows [5]:

$$
\begin{aligned}
& L_{g} L_{f}^{k} h(x)=0, \\
& L_{g} L_{f}^{r-1} h(x) \neq 0,
\end{aligned}
$$

Utilizing (12), the relative degree of the system (10) can be obtained as $r=2$. Hence, the switching surface can be designed as

$$
s(t)=\left(\frac{d}{d t}+\lambda\right)^{r-1} e(t)=\dot{e}(t)+\lambda e(t),
$$

where $e(t)=\alpha-\alpha_{d}$ and $\lambda$ is strictly positive constant defining the bandwidth of the error dynamics. Differentiating $s$, we have

$$
\begin{gathered}
\dot{s}(t)=\nu(x, t)+b \delta_{c}, \\
\nu(x, t)=\lambda \dot{e}-\ddot{\alpha}_{d}+\dot{q}+K_{\alpha} M\left[\frac{\partial}{\partial \alpha}\left[C_{N} \cos (\alpha)\right] \dot{\alpha}-\frac{d_{n}}{\tau_{\alpha}} \delta\right], \\
b=K_{\alpha} M \frac{d_{n}}{\tau_{\alpha}} .
\end{gathered}
$$

In (14) derivatives of the desired angle-of-attack, $\alpha_{d}$, generated by guidance subsystem can be obtained by conventional numerical approaches and there would be no measurement noise which is common for navigation sensors. The sliding mode autopilot design is then choosing the control input such that the following attractive equation is satisfied:

$$
\frac{1}{2} \frac{d}{d t} s^{T} s \leq-\rho|s|
$$

where $\rho$ is strictly positive constant that determines the desired reaching time to the sliding surface. The attractive equation (15) is also called sliding condition and implies that the distance to the sliding surface decreases along all system trajectories. Furthermore, the sliding condition makes the sliding surfaces an invariant set; that is, once a system trajectory reaches the surface, it will remain on it for the rest of time. In addition, for any initial condition, the sliding surface will be reached in a finite time. When (14) is used, the controller that satisfies the sliding conditions (15) can be chosen as

$$
\delta_{c}=b^{-1}[-v(x, t)-\rho \operatorname{sign}(s)] .
$$

This control can be viewed as consisting of two parts: the term $-b^{-1} v(x, t)$, called equivalent control, which guaranties $\dot{s}(t)=0$ for the nominal model, and the other term $-b^{-1} \rho \operatorname{sign}(s)$, incorporated to deal with uncertainties. However, because of the discontinuity across the sliding surfaces, the preceding control law may result in control chattering. As a practical matter, chattering is undesirable phenomena because it involves very high control action and may excite high-frequency dynamics neglected in the modeling. The discontinuity in the control law can be dealt with by defining a thin boundary layer of width $\Phi$ around the sliding surface, that is, replacing sign(s) with continuous saturation functions $\operatorname{sat}(s / \Phi)$, where $\operatorname{sat}(x)=$ $x$ if $|x| \leq 1$ and $\operatorname{sat}(x)=\operatorname{sign}(x)$ otherwise. Thus, our sliding controller is modified as follows:

$$
\delta_{c}=b^{-1}\left[-v(x, t)-\rho \operatorname{sat}\left(\frac{s}{\Phi}\right)\right] .
$$




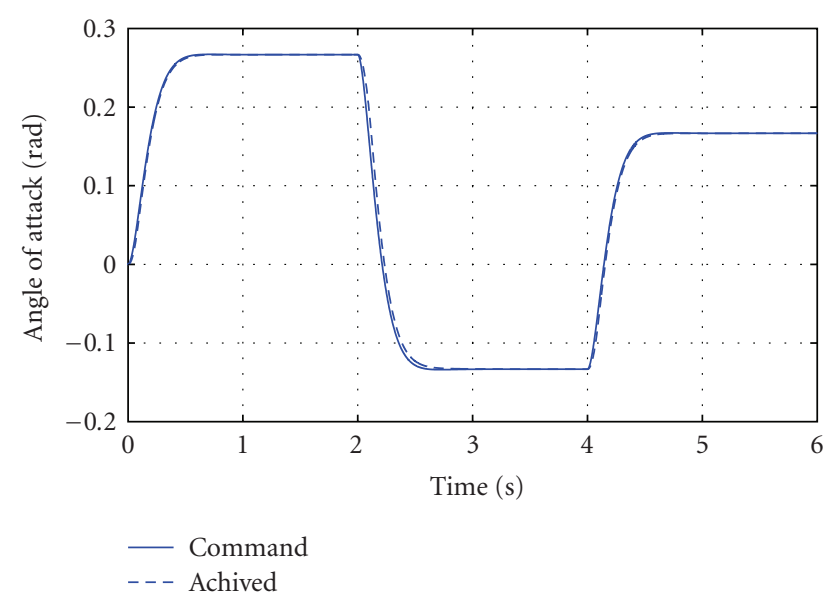

FIGURE 2: Vehicle response to a sequence of step command.

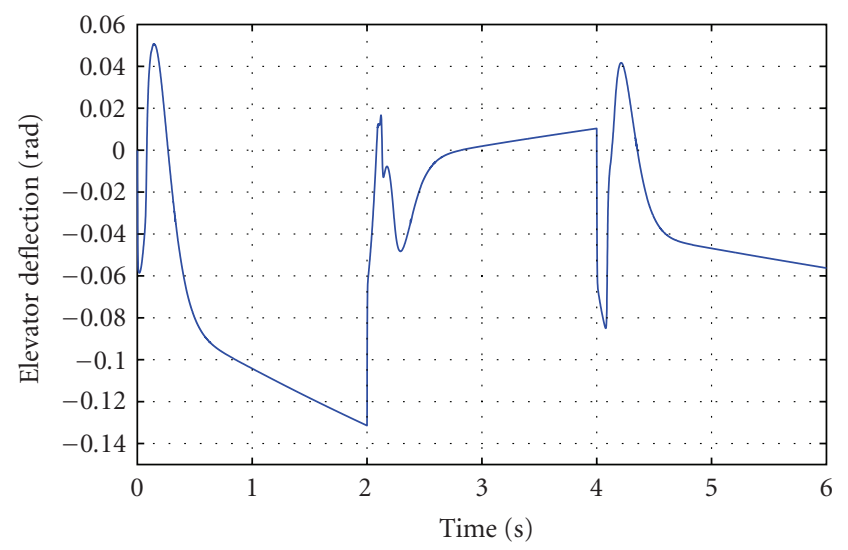

FIgURE 3: Elevator deflection versus time.

Figures 2 and 3 show the response of the nominal model, without parameter uncertainty. In the simulation studies for the nominal model, $\rho$ is taken as 10 and $\Phi$ is chosen as 0.05 . Figure 4 shows the simulation results for the same maneuver albeit in the four various possible parameter uncertainties. The results show that robustness and stability have indeed been achieved. The price paid to achieve robustness in this case is in the form of large gain $\rho$ and control chattering. However, the control gain cannot be chosen arbitrary large due to practical considerations such as reaching control surface deflection limits. Furthermore, control chattering is undesirable in practice because it involves high control activity and may excite high-frequency unmodeled dynamics. In simulation, the width of the boundary layer has expanded to reduce chattering. To eliminate chattering, the width of the boundary layer has to be further expanded, which will result in large steady-state errors. To circumvent this drawback an adaptation mechanism will be utilized in sequel.

\section{Adaptive Sliding Mode Control Design}

The sliding mode controller exhibits good tracking in the presence of parametric uncertainty at the expense of high

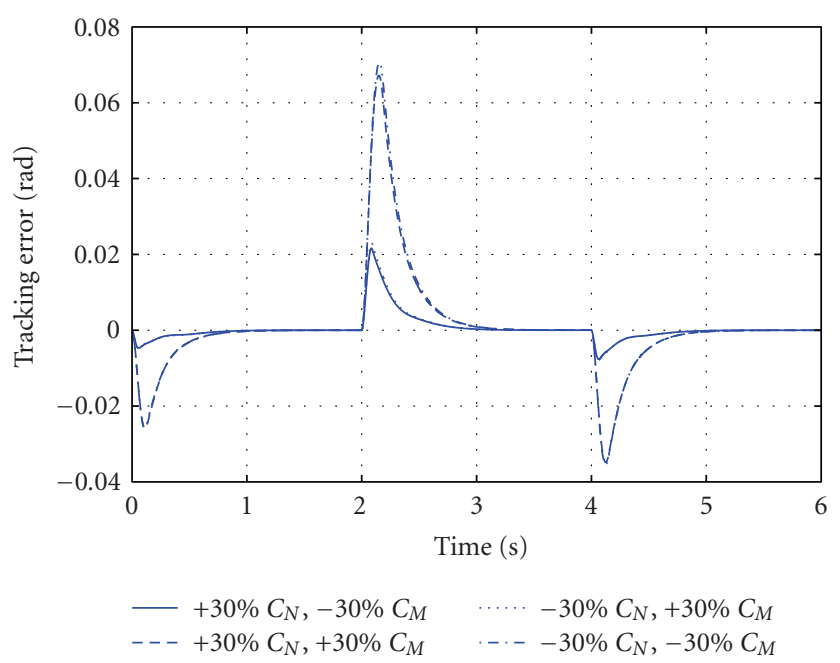

FIGURE 4: Tracking error for various possible parameter uncertainties.

gains and control chattering. The performance of the sliding mode controller is dependent on the size of the parametric uncertainties involved. An undesirable characteristic of this controller is the quick erosion of performance to gain robustness. This shortcoming of the sliding controller has urged combining the given control law with online parameter adaptation. In this section, an adaptive sliding mode controller will be developed that combines an online parameter estimator with the sliding mode controller of the preceding section. The adaptive laws for updating parameters are generated commonly using the Lyapunov approach. The dynamics of the system is more sensitive to the uncertainty parameters in the aerodynamic coefficients achieved by wind-tunnel measurements. $C_{N}$ embodies parameters $\beta_{i n}, d_{n}$ and $C_{M}$ embodies parameters $\beta_{i m}, d_{m}$, and $i=1,2,3$. To deal with the parametric uncertainty, we will combine the sliding mode controller with an online parameter estimator forming an adaptive sliding mode controller:

$$
\delta_{c}=\hat{b}^{-1}\left[-\hat{\nu}(x, t)-\rho \operatorname{sat}\left(\frac{s}{\Phi}\right)\right]
$$

where

$$
\begin{aligned}
\hat{v}(x, t)= & \lambda \dot{e}-\ddot{\alpha}_{d}+K_{q} M^{2} \hat{C}_{M} \\
& +K_{\alpha} M\left[\frac{\partial}{\partial \alpha}\left[\hat{C}_{N} \cos (\alpha)\right] \dot{\alpha}-\frac{\hat{d}_{n}}{\tau_{\alpha}} \delta\right], \\
\hat{b}= & K_{\alpha} M \frac{\hat{d}_{n}}{\tau_{\alpha}} \\
\hat{C}_{N}= & \hat{\beta}_{1 n} \alpha^{3}+\hat{\beta}_{2 n} \alpha|\alpha|+\hat{\beta}_{3 n} \alpha+\hat{d}_{n} \delta \\
\hat{C}_{M}= & \hat{\beta}_{1 m} \alpha^{3}+\hat{\beta}_{2 m} \alpha|\alpha|+\hat{\beta}_{3 m} \alpha+\hat{d}_{m} \delta .
\end{aligned}
$$




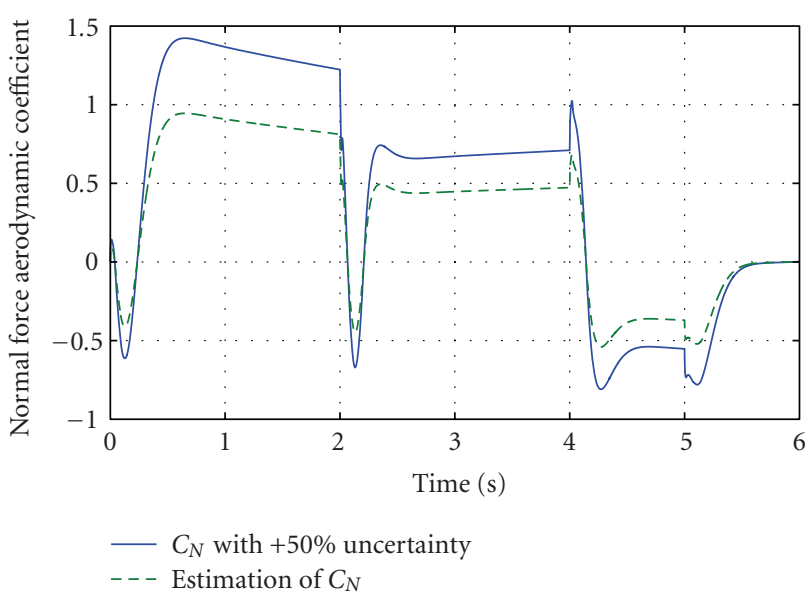

Figure 5: Nominal force aerodynamic coefficient estimation with adaptive scheme.

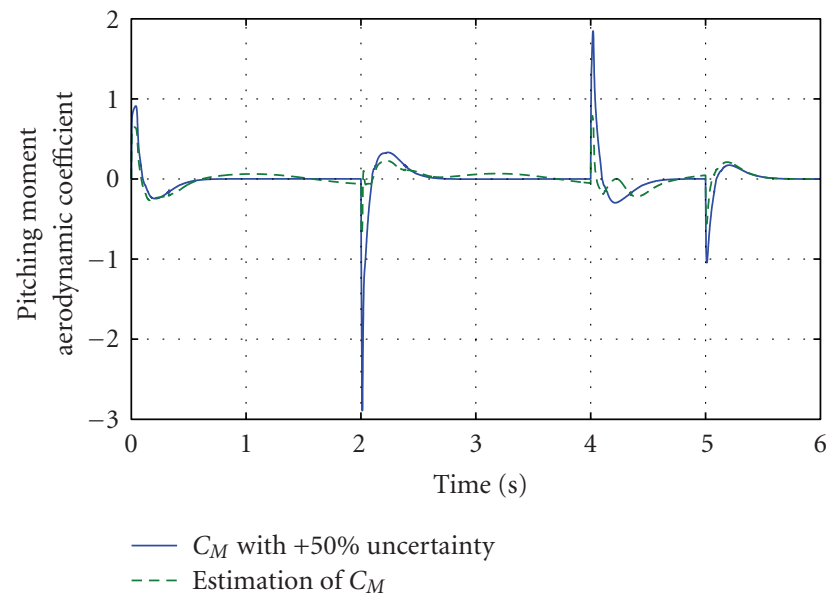

Figure 6: Pitching moment aerodynamic coefficient estimation with adaptive scheme.

In order to proceed with adaptive parameter estimation for aerodynamic coefficients we will follow as

$$
\begin{aligned}
& \hat{C}_{N}(\alpha, \delta)=\hat{P}_{n}^{T} W_{n}, \\
& \widehat{C}_{M}(\alpha, \delta)=\hat{P}_{m}^{T} W_{m},
\end{aligned}
$$

where

$$
\begin{gathered}
\hat{P}_{n}=\left[\begin{array}{c}
\hat{\beta}_{1 n} \\
\hat{\beta}_{2 n} \\
\hat{\beta}_{3 n} \\
\hat{d}_{n}
\end{array}\right], \quad \hat{P}_{m}=\left[\begin{array}{c}
\hat{\beta}_{1 m} \\
\hat{\beta}_{2 m} \\
\hat{\beta}_{3 m} \\
\hat{d}_{m}
\end{array}\right], \\
W_{n}=W_{m}=\left[\begin{array}{c}
\alpha^{3} \\
\alpha|\alpha| \\
\alpha \\
\delta
\end{array}\right] .
\end{gathered}
$$

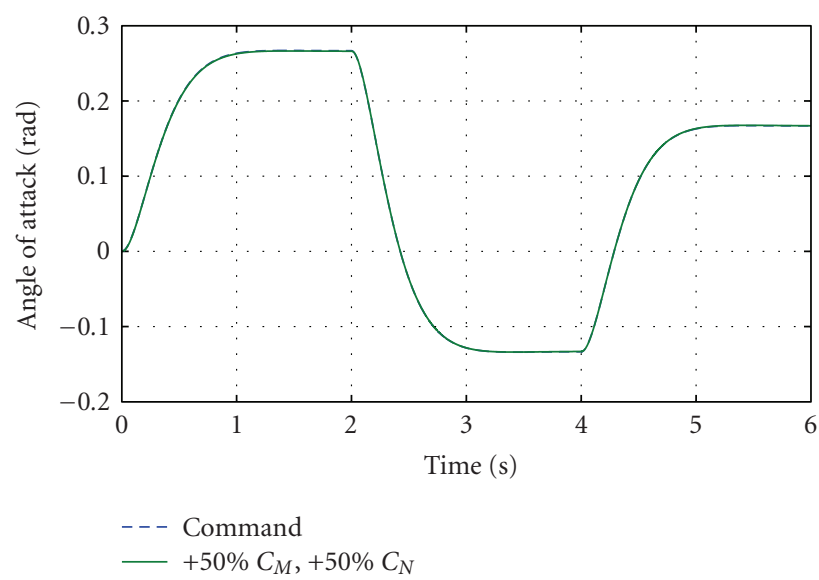

Figure 7: Angle of attack for a sequence of step command with parameter variation.

The switching dynamics based on the control input (18) can be written as

$$
\dot{s}+k \cdot \operatorname{sat}\left(\frac{s}{\Phi}\right)=-K_{q} M^{2} \widetilde{P}_{m}^{T} W_{m}+\widetilde{P}_{n}^{T} V_{n}
$$

where $\widetilde{C}_{N}=\hat{C}_{N}-C_{N}, \widetilde{C}_{M}=\hat{C}_{M}-C_{M}$, and $V_{n}$ is the resulting regression vector:

$$
V_{n}=\left[\begin{array}{c}
K_{\alpha}\left(\alpha^{3} M \dot{\alpha} \sin \alpha-3 \alpha^{2} \dot{\alpha} M \cos \alpha\right) \\
K_{\alpha}(\alpha|\alpha| M \dot{\alpha} \sin \alpha-2 \alpha|\alpha| \dot{\alpha} M \cos \alpha) \\
K_{\alpha}(\alpha M \dot{\alpha} \sin \alpha-\dot{\alpha} M \cos \alpha) \\
K_{\alpha} \delta M \dot{\alpha} \sin \alpha
\end{array}\right] .
$$

The adaptive laws can be derived using the Lyapunov synthesis. Consider the Lyapunov-like function:

$$
V=\frac{1}{2} s^{T} s+\frac{1}{2} \Gamma^{-1}\left(\widetilde{P}_{m}^{T} \widetilde{P}_{m}+\widetilde{P}_{n}^{T} \widetilde{P}_{n}\right)
$$

where $\widetilde{P}_{n}=\hat{P}_{n}-P_{n}, \widetilde{P}_{n}=\hat{P}_{n}-P_{n}$, and $\Gamma$ is a definite positive scalar. With the assumption that the variation of $\beta_{i n}, \beta_{i m}, d_{n}, d_{m}$ is small, (i.e., $\dot{P}_{n} \approx 0$ and $\dot{P}_{m} \approx 0$, the following can be deduced:

$$
\begin{gathered}
\dot{\hat{P}}_{n}=-\Gamma s V_{n}, \\
\dot{\hat{P}}_{m}=\Gamma s K_{q} M^{2} W_{m} .
\end{gathered}
$$

Performance of parameter estimations based on the achieved dynamics in (25) has been demonstrated in Figures 5 and 6. The flight control behavior under the parameters variation has been shown in Figures 7, 8, and 9. 


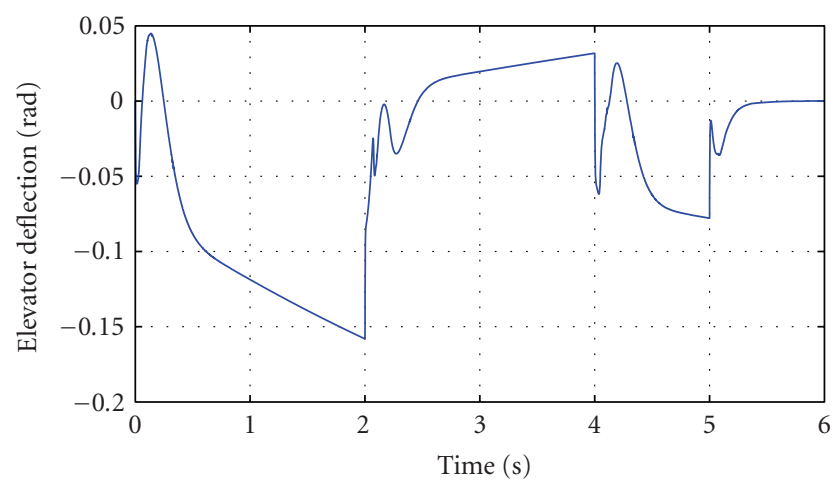

FIgURE 8: Elevator deflection with adaptive scheme.

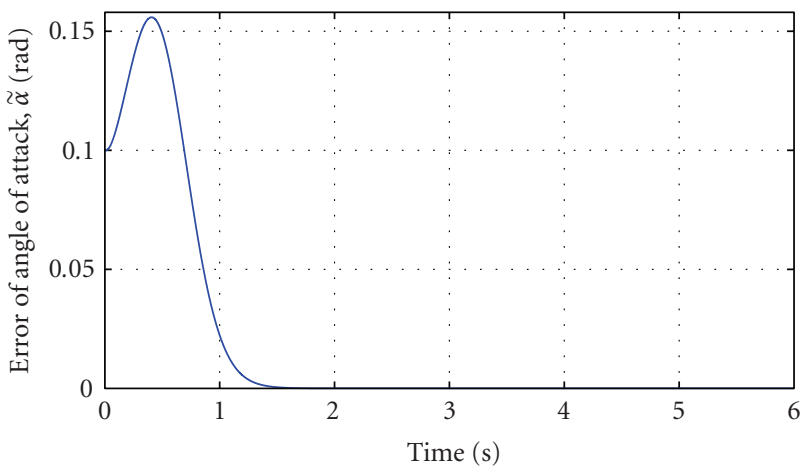

FIGURE 9: Tracking error for observer $\tilde{\alpha}=\hat{\alpha}-\alpha$.

\section{Sliding Mode Observer-Control Design}

The sliding mode controller developed in the preceding sections assumes that full states are available for measurement. In practice, however, only the states that correspond to pitch rate $q$ are expected to be measured. The state variables corresponding to the angle-of-attack $\alpha$ may be difficult to measure in an actual supersonic flight vehicle. Accurate measurements are costly and difficult if at all possible to obtain practically. In this section, a sliding mode observer is designed that can provide estimation of the angle-ofattack based on the measurement of pitch rate. When the techniques developed in [6-8] are applied, a sliding mode observer is designed whose structure is given by

$$
\begin{gathered}
\dot{\hat{q}}(t)=K_{q} M^{2} \hat{C}_{M}-\eta_{1} \tilde{q}-k_{1} \operatorname{sign}(\tilde{q}), \\
\dot{\hat{\alpha}}(t)=K_{\alpha} M \hat{C}_{N} \cos (\hat{\alpha})+\hat{q}-\eta_{2} \tilde{q}-k_{2} \operatorname{sign}(\tilde{q}), \\
\tilde{q}=\hat{q}-q,
\end{gathered}
$$

where $\widehat{C}_{N}(\hat{\alpha}, \delta)=\beta_{1 n} \hat{\alpha}^{3}+\beta_{2 n} \hat{\alpha}|\hat{\alpha}|+\beta_{3 n} \hat{\alpha}+d_{n} \delta$ and $\widehat{C}_{M}(\hat{\alpha}, \delta)=$ $\beta_{1 m} \hat{\alpha}^{3}+\beta_{2 m} \hat{\alpha}|\hat{\alpha}|+\beta_{3 m} \hat{\alpha}+d_{m} \delta$.

$\eta_{1}$ and $\eta_{2}$ are positive constants selected as 20 and 0.6. $k_{1}$ and $k_{2}$ are sliding gains and chosen as 0.0001 and 0.001 ,

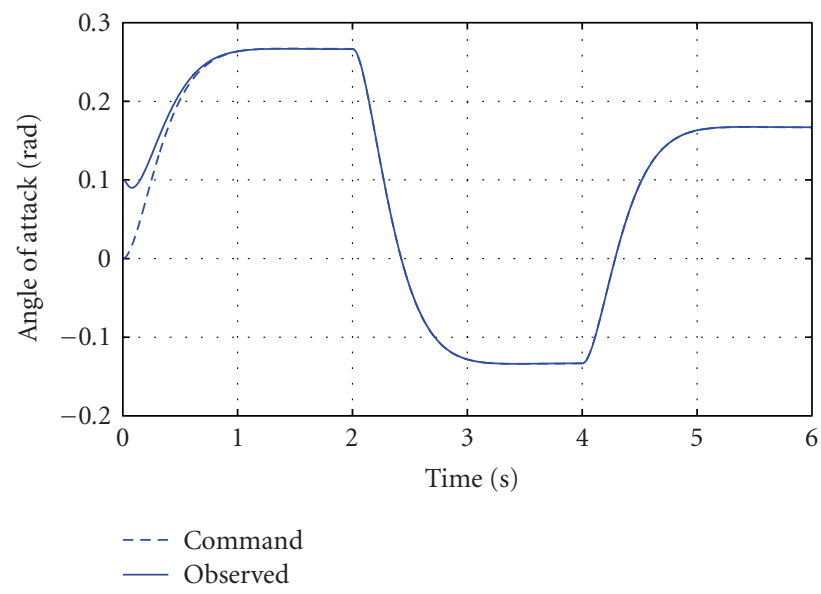

Figure 10: Output tracking with observer.

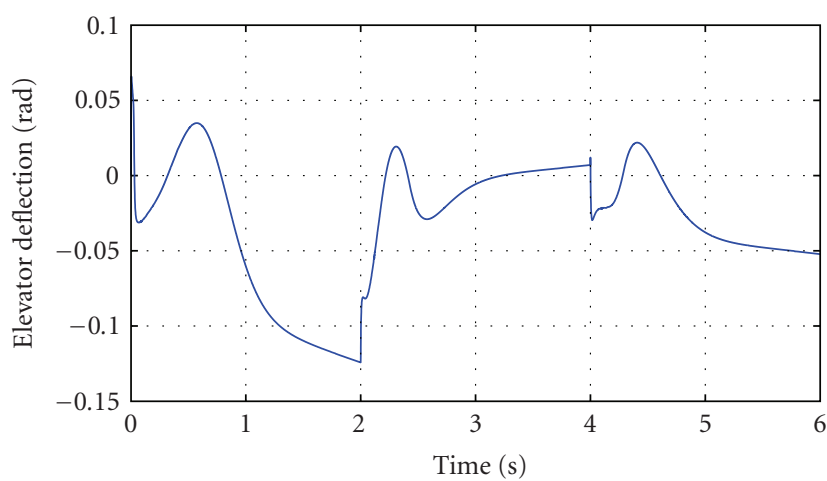

FIGURE 11: Input control with observer.

respectively. The sliding surface of the observer is defined by $s_{o}=\tilde{q}=0$. Using the same procedure as in the preceding section the dynamics of the observer can be obtained as

$$
\dot{\tilde{\alpha}}=-\frac{k_{2}}{k_{1}} K_{q} M^{2}\left(\widehat{C}_{M}-C_{M}\right)+K_{\alpha}\left[\hat{C}_{N} \cos \hat{\alpha}-C_{N} \cos \alpha\right]
$$

The control input then will be conducted as

$$
\delta_{c}=b^{-1}[-\hat{\nu}(x, t)-\rho \operatorname{sign}(s)]
$$

where $b=K_{\alpha} M\left(d_{n} / \tau_{\alpha}\right)$ and

$$
\begin{aligned}
\hat{\nu}(x, t)= & \lambda \dot{e}-\ddot{\alpha}_{d}+K_{q} M^{2} \widehat{C}_{M} \\
& +K_{\alpha} M\left[\frac{\partial}{\partial \hat{\alpha}}\left[\hat{C}_{N} \cos (\hat{\alpha})\right] \dot{\hat{\alpha}}-\frac{d_{n}}{\tau_{\alpha}} \delta\right] .
\end{aligned}
$$




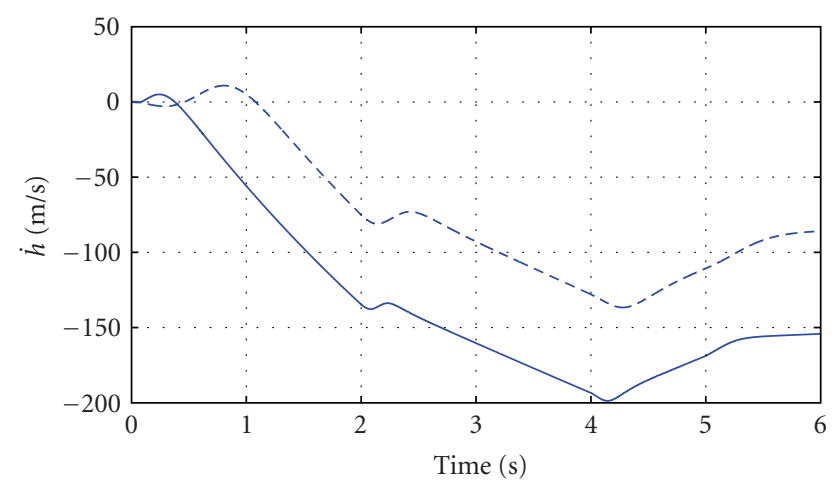

— Without observer

- - - With observer

FIgURE 12: Climb rate, $\dot{h}$, with/without observer.

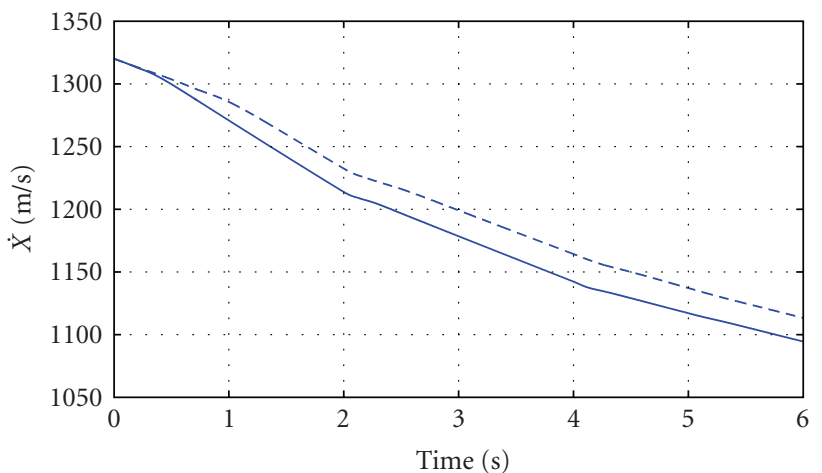

- Without observer

- - - With observer

FIGURE 13: Horizontal speed, $\dot{X}$, with/without observer.

Performance of the observer for estimation of the angleof-attack has been illustrated in Figure 9. By employing observed angle-of-attack from designed sliding mode observer and utilizing the tracking error as $e(t)=\left(\hat{\alpha}-\alpha_{d}\right)$, output tracking of the system, control input, climb rate, and horizontal speeds can be achieved for the nominal system as shown through Figures 10, 11, 12, to Figure 13, respectively, where the initial condition for the observer has been considered as $\hat{\alpha}(t=0)=0.1 \mathrm{rad}$. Figure 14 shows the simulation results for four various possible parameter variations in the system that influences performance of the observer. The results show that robustness and stability have been achieved.

\section{Adaptive Sliding Mode Observer-Control Design}

In this section adaptive parameter estimation will be conducted on the sliding mode observer designed in the preceding section. This will inhibit performance decrease against parameter variation introduced by $C_{N}$ and $C_{M}$. Now

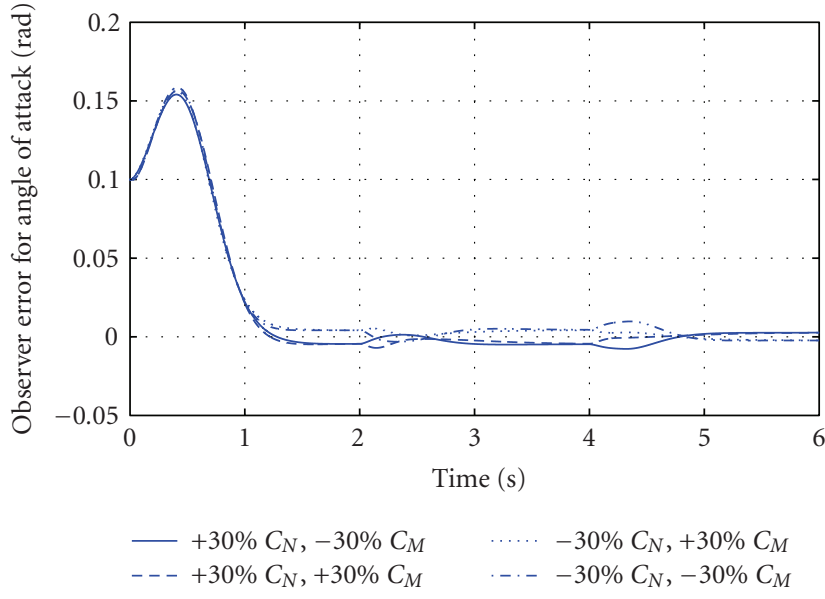

FIgURE 14: Tracking error for observer in presence of various parameter variations.

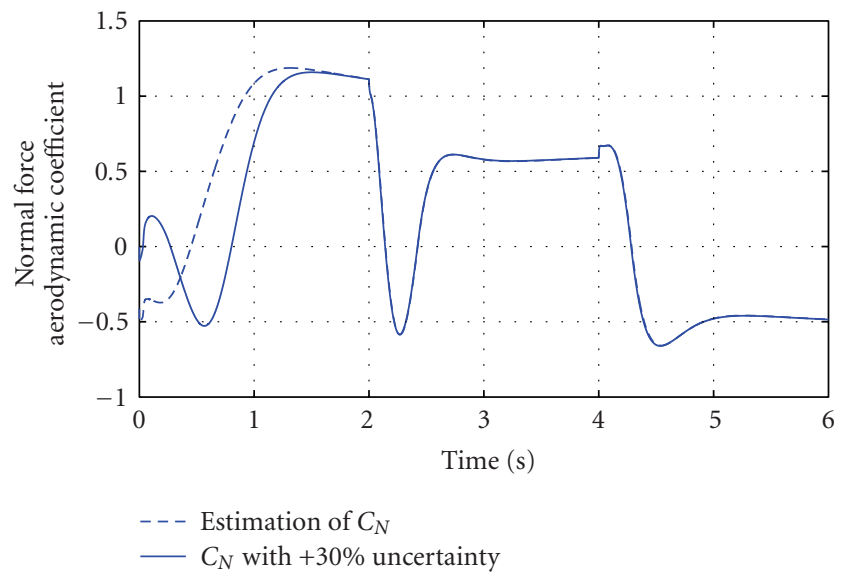

FIGURE 15: Normal force aerodynamic coefficient estimation with adaptive sliding observer-control.

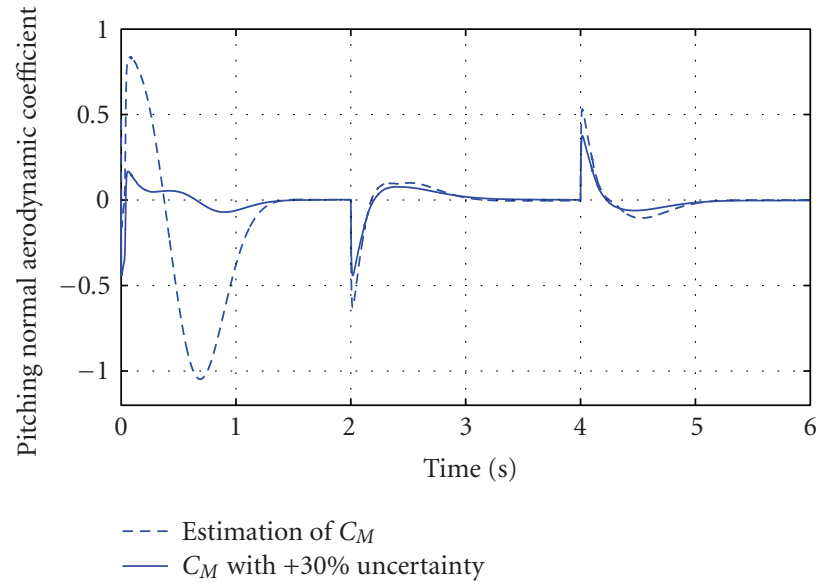

FIGURE 16: Pitching moment aerodynamic coefficient estimation with adaptive sliding observer-control. 


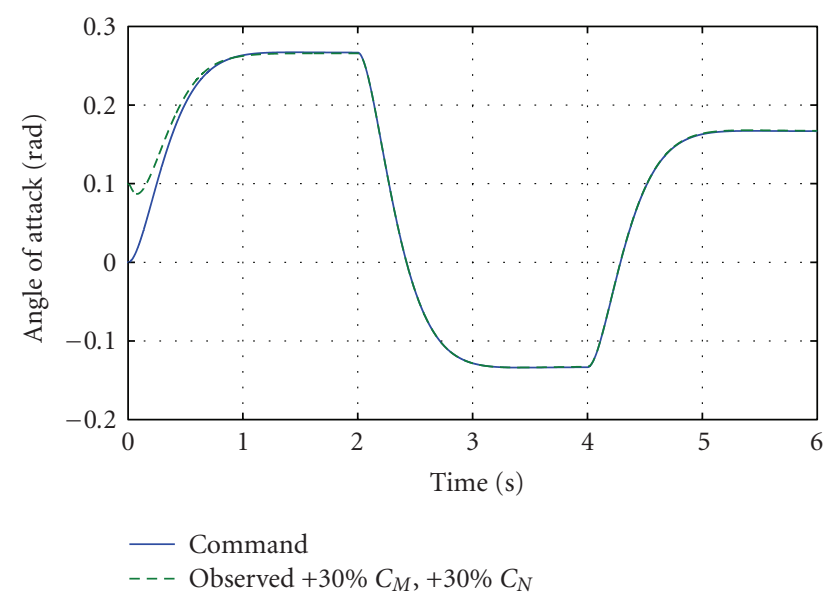

FIGURE 17: Output tracking with adaptive sliding observer-control.

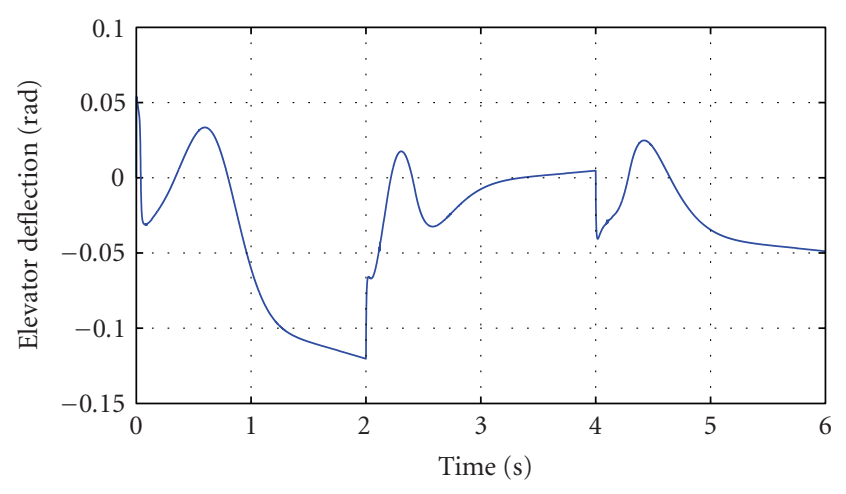

FIGURE 18: Input control with adaptive sliding observer-control.

(20) can be rewritten as

$$
\begin{aligned}
& \widehat{C}_{N}(\hat{\alpha}, \delta)=\hat{P}_{n}^{T} \widehat{W}_{n}, \\
& \widehat{C}_{M}(\widehat{\alpha}, \delta)=\widehat{P}_{m}^{T} \widehat{W}_{m},
\end{aligned}
$$

where

$$
\begin{gathered}
\hat{P}_{n}=\left[\begin{array}{c}
\hat{\beta}_{1 n} \\
\hat{\beta}_{2 n} \\
\hat{\beta}_{3 n} \\
\hat{d}_{n}
\end{array}\right], \quad \hat{P}_{m}=\left[\begin{array}{c}
\hat{\beta}_{1 m} \\
\hat{\beta}_{2 m} \\
\hat{\beta}_{3 m} \\
\hat{d}_{m}
\end{array}\right], \\
\widehat{W}_{n}=\widehat{W}_{m}=\left[\begin{array}{c}
\hat{\alpha}^{3} \\
\hat{\alpha}|\hat{\alpha}| \\
\hat{\alpha} \\
\delta
\end{array}\right] .
\end{gathered}
$$

Consider Lyapunov synthesis as

$$
V=\frac{1}{2}(\widetilde{q})^{2}+\frac{1}{2} \Gamma^{-1}\left(\widetilde{P}_{m}^{T} \widetilde{P}_{m}+\widetilde{P}_{n}^{T} \widetilde{P}_{n}\right) .
$$

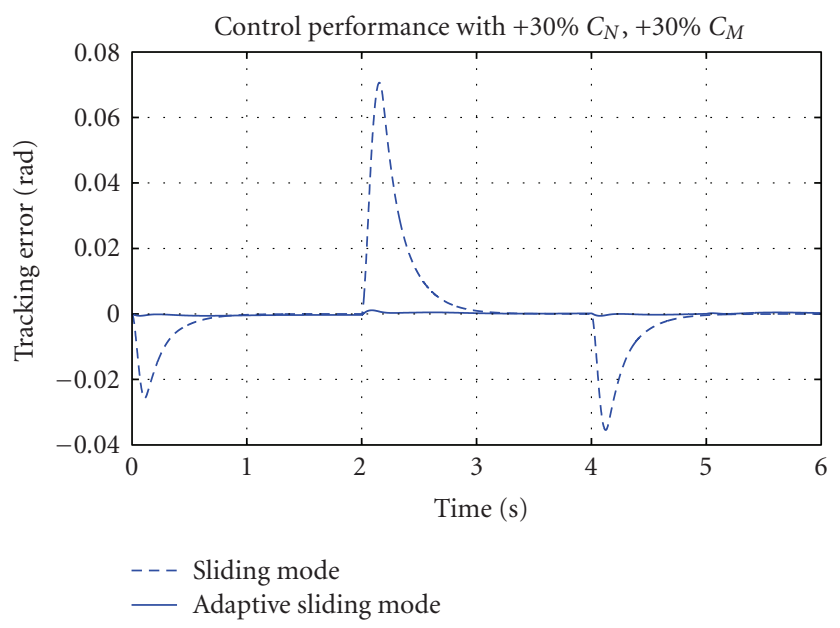

FIGURE 19: Tracking error with sliding mode and adaptive sliding mode.

With the assumption that the variation of $\beta_{i n}, \beta_{i m}, d_{n}, d_{m}$ is small (i.e., $\dot{P}_{n} \approx 0$ and $\dot{P}_{m} \approx 0$ ), the following can be deduced

$$
\begin{aligned}
& \dot{\hat{P}}_{n j}=\frac{\widetilde{P}_{n j}^{-1} \Gamma}{4 \tilde{q}\left(\dot{\tilde{q}}-K_{q} M^{2} P_{m}^{T} \widehat{W}_{m}\right)}, \\
& \dot{\hat{P}}_{m j}=-\frac{\Gamma}{4 K_{q} M^{2} \tilde{q} \widehat{W}_{m j}} .
\end{aligned}
$$

Performance of parameter estimations based on the achieved dynamics in (33) has been demonstrated in Figures ?? and 16. The flight control behavior under the parameters variation has been shown in Figures 17 and 18. Performance of the adaptive sliding mode control-observer demonstrated both for the nominal and perturbed system accordingly. Output tracking errors for the perturbed system following the designed approaches are shown in Figure 19 with robustness against parameters uncertainties.

\section{Conclusion}

A robust control is designed for a hypothetical nonlinear supersonic flight vehicle using sliding mode and adaptive control theories based on the Lyapunov theory. First a robust sliding mode control is designed based on the assumption that all system states are available. An adaptation mechanism is then integrated with the robust control to circumvent large parameters uncertainties and decrease both chattering phenomena and boundary layer thickness expansion which exhibits large steady output tracking error. In order to deal with shortcomings arising from system states unavailability a sliding mode observer is developed to estimate angleof-attack which is difficult to measure in practice. The sliding mode observer finally is integrated with an adaptation scheme that makes system to be adaptive against large parameter variations. 


\section{Nomenclature}

\begin{tabular}{|c|c|}
\hline$C_{D}:$ & $\begin{array}{l}\text { Axial force dimensionless } \\
\text { aerodynamic }\end{array}$ \\
\hline$C_{N}:$ & $\begin{array}{l}\text { Normal force dimensionless } \\
\text { aerodynamic polynomial }\end{array}$ \\
\hline$C_{M}:$ & $\begin{array}{l}\text { Pitching moment dimensionless } \\
\text { aerodynamic polynomial }\end{array}$ \\
\hline$d:$ & Reference diameter, $\mathrm{m}$ \\
\hline$F_{x}:$ & Axial force, $\mathrm{N}$ \\
\hline$F_{z}:$ & Normal force, $\mathrm{N}$ \\
\hline$g:$ & Gravity, $\mathrm{m} / \mathrm{s}^{2}$ \\
\hline$h:$ & Altitude, $\mathrm{m}$ \\
\hline$I_{y}:$ & Moment of inertia, $\mathrm{kg} \mathrm{m}^{2}$ \\
\hline$K_{\alpha}, K_{q}:$ & Vehicle constant parameters \\
\hline$m:$ & Vehicle mass, $\mathrm{kg}$ \\
\hline M: & Mach number \\
\hline$M_{y}:$ & Pitching moment, $\mathrm{N} \cdot \mathrm{m}$ \\
\hline$P_{o}:$ & Static pressure, $\mathrm{N} / \mathrm{m}^{2}$ \\
\hline$q:$ & Pitch rate, $\mathrm{rad} / \mathrm{s}$ \\
\hline Q: & Dynamic pressure, $\mathrm{kg} / \mathrm{m} \mathrm{s}^{2}$ \\
\hline$S:$ & Reference surface area, $\mathrm{m}^{2}$ \\
\hline$v_{s}:$ & Speed of sound, m/s \\
\hline$V_{v}:$ & Speed of vehicle, $\mathrm{m} / \mathrm{s}$ \\
\hline$\alpha:$ & Angle-of-attack, rad \\
\hline$\beta_{1 n}, \beta_{2 n}, \beta_{3 n}:$ & $\begin{array}{l}\text { Angle of attack component of normal } \\
\text { force dimensionless aerodynamic } \\
\text { polynomial coefficients }\end{array}$ \\
\hline$\beta_{1 m}, \beta_{2 m}, \beta_{3 m}:$ & $\begin{array}{l}\text { Angle of attack component of } \\
\text { pitching moment dimensionless } \\
\text { aerodynamic polynomial coefficients }\end{array}$ \\
\hline$\delta:$ & Actual tail fin deflection, rad \\
\hline$\delta_{c}:$ & Commanded tail fin deflection, rad \\
\hline$\theta:$ & Pitch angle, rad \\
\hline$\tau_{a}:$ & Tail actuator time constant, sec. \\
\hline
\end{tabular}

\section{References}

[1] A. R. Mehrabian and J. Roshanian, "Skid-to-turn missile autopilot design using scheduled eigenstructure assignment technique," Proceedings of the Institution of Mechanical Engineers $G$, vol. 220, no. 3, pp. 225-239, 2006.

[2] R. A. Nichols, R. T. Reichert, and W. J. Rugh, "Gain scheduling for H-infinity controllers: a flight control example," IEEE Transactions on Control Systems Technology, vol. 1, no. 2, pp. 6978, 1993.

[3] J. J. Zhu and M. C. Mickle, "Missile autopilot design using a new linear time-varying control technique," Journal of Guidance, Control, and Dynamics, vol. 20, no. 1, pp. 150-157, 1997.

[4] US Standard Atmosphere, US government printing office, Washington, DC, USA, 1976.

[5] A. Isidori, Nonlinear Control Systems, Springer, New York, NY, USA, 1995.

[6] J.-J. E. Slotine, J. K. Hedrick, and E. A. Misawa, "On sliding observers for nonlinear systems," ASME Journal of Dynamics Systems and Control, vol. 109, pp. 245-252, 1987.
[7] I. Haskara, U. Ozguner, and V. Utkin, "On variable structure observers," in Proceedings of the IEEE International Workshop on Variable Structure Systems (VSS '96), pp. 193-198, December 1996.

[8] V. I. Utkin, J. Guldner, and J. Shi, Sliding Modes in Electromechanical Systems, Taylor \& Francis, London, UK, 1998. 

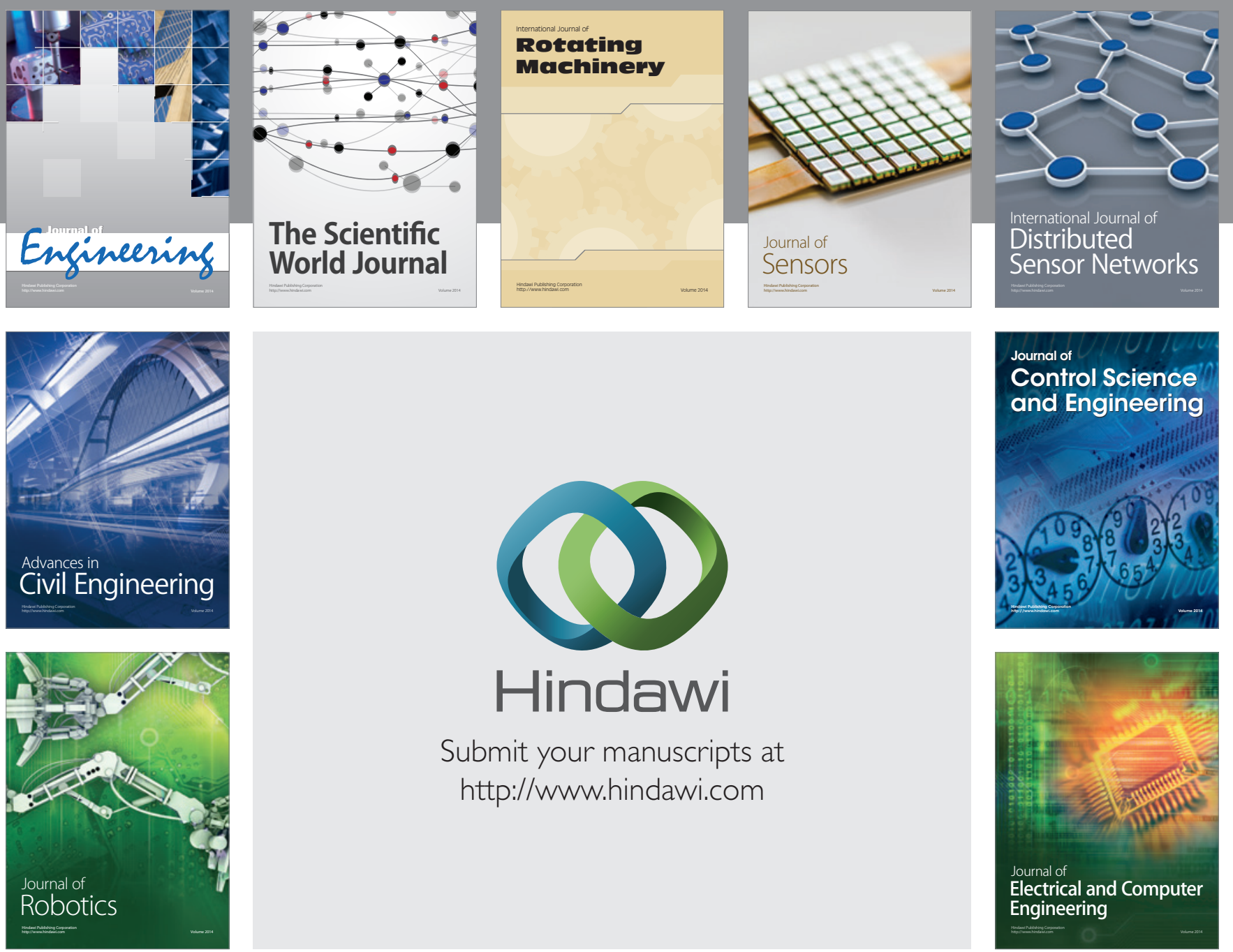

Submit your manuscripts at

http://www.hindawi.com
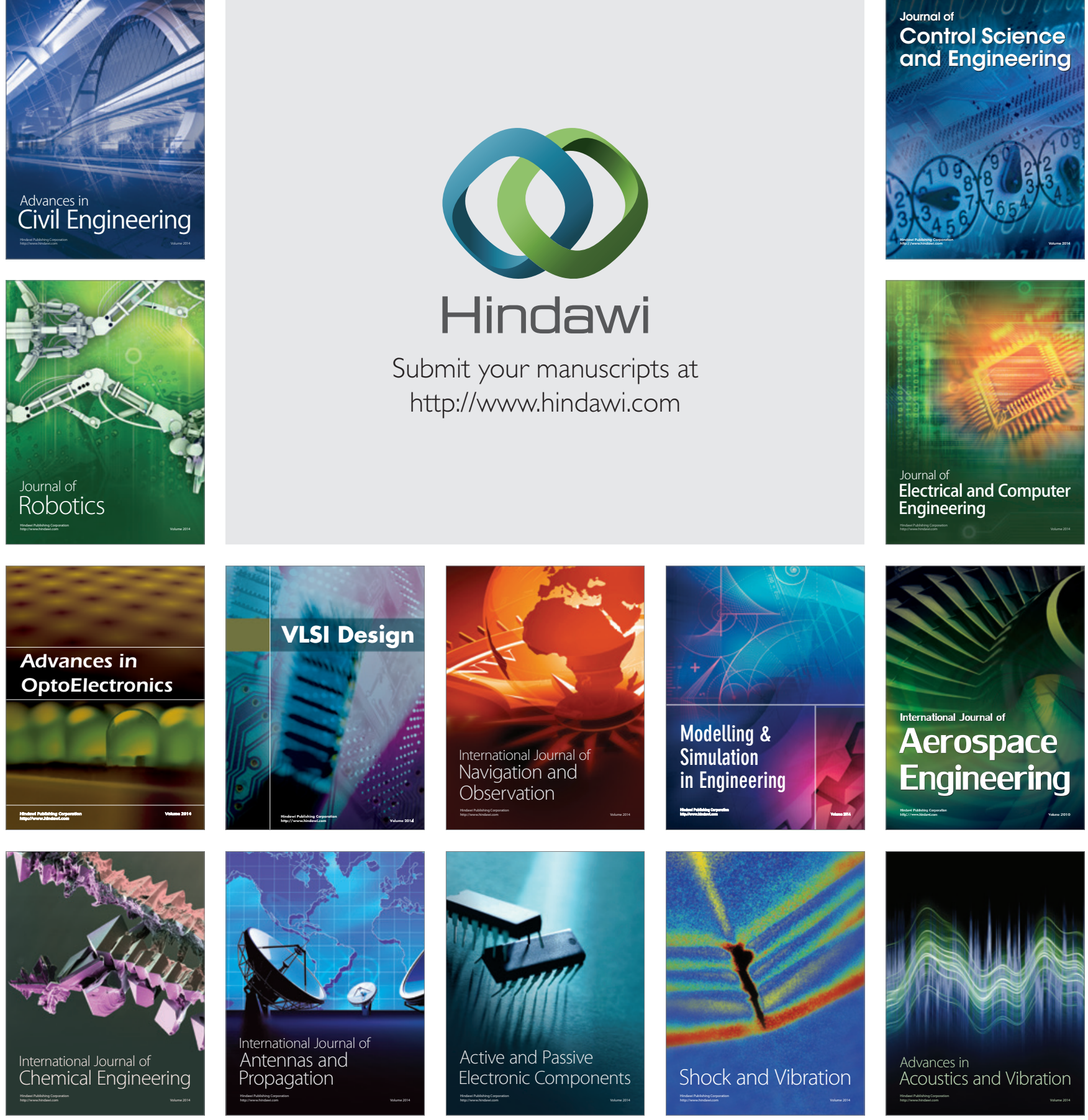